\title{
A trombose venosa profunda pós-COVID-19 e seu manejo farmacológico
}

\author{
Post-COVID-19 deep vein thrombosis and its pharmacological management
}

Trombosis venosa profunda post-COVID-19 y su manejo farmacológico

Clara Maria Muguet

ORCID: https://orcid.org/0000-0002-7001-2192

Universidade Estácio de Sá, Brasil

E-mail: claracostamuguet7@gmail.com

Karoline Guimarães

ORCID: https://orcid.org/0000-0001-5092-9406

Universidade Estácio de Sá, Brasil

E-mail: karolguimaraess21@gmail.com

Lilian Bastos

ORCID: https://orcid.org/0000-0002-8683-5273

Universidade Estácio de Sá, Brasil

E-mail: lilian.bastos1997@gmail.com

Marcela Baroni

ORCID: https://orcid.org/0000-0002-3232-7601

Universidade Estácio de Sá, Brasil

E-mail: marcelacbaroni@gmail.com

Marcela Curty

ORCID: https://orcid.org/0000-0002-0695-5719

Universidade Estácio de Sá, Brasil

E-mail: marcelacurty12@hotmail.com

Marcos Marzano

ORCID: https://orcid.org/0000-0001-8486-6407

Universidade Estácio de Sá, Brasil

E-mail: marcosmarzano@hotmail.com

Mariana Costa

ORCID: https://orcid.org/0000-0002-2512-3248

Universidade Estácio de Sá, Brasil

E-mail: mari.marianacostaaa@gmail.com

Mirela Tardelli

ORCID: https://orcid.org/0000-0003-0237-6776

Universidade Estácio de Sá, Brasil

E-mail: mirelatardellimed@gmail.com

Rafael Risi

ORCID: https://orcid.org/0000-0002-4264-7465

Universidade Estácio de Sá, Brasil

E-mail: rafaelrisi@gmail.com

Renata Lustosa

ORCID: https://orcid.org/0000-0001-5617-4307

Universidade Estácio de Sá, Brasil

E-mail: re_lustosa@hotmail.com

Vanessa Estato

ORCID: https://orcid.org/0000-0002-1581-9620

Universidade Estácio de Sá, Brasil Instituto Oswaldo Cruz, Brasil

E-mail: vanessaestato@gmail.com

\begin{abstract}
Resumo
A infecção causada pelo coronavírus SARS-CoV-2 apresenta casos clínicos que variam de assintomáticos para graves. Mesmo nos casos mais brandos, podem ser observadas sequelas relacionadas a um estado hipercoagulável associado à COVID-19, com a prevalência aumentada de Tromboembolismo Venoso (TEV). A ocorrência de TEV é maior principalmente em pacientes internados em unidades de terapia intensiva e requer terapia de anticoagulação, no entanto, a trombogênse pós-COVID também pode ocorrer numa fase mais tardia, na ausência de carga viral. Os mecanismos pró-trombóticos relacionados ao SARS-CoV-2 apresentam divergências no que se refere ao uso de medicamentos antiplaquetários e anticoagulantes em pacientes com suspeita ou confirmação de COVID-19. A farmacologia e a aplicabilidade clínica dos fármacos anticoagulantes como as heparinas e os anticoagulantes orais, serão descritas neste estudo. Este estudo é um estudo exploratório com o objetivo de abordar as questões relacionadas
\end{abstract}


ao tratamento farmacológico e os mecanismos de ação dos fármacos até então recomendados para o tratamento e profilaxia dos eventos trombóticos em pacientes acometidos pela COVID-19.

Palavras-chave: SARS-CoV-2; COVID-19; Anticoagulantes; Tromboembolia venosa.

\begin{abstract}
Infection caused by the SARS-CoV-2 coronavirus presents clinical cases that range from asymptomatic to severe. Even in milder cases, sequelae related to a hypercoagulable state associated with COVID-19 can be observed, with an increased prevalence of Venous Thromboembolism (VTE). The occurrence of VTE is higher in patients, mainly patients hospitalized in intensive care units and requires anticoagulation therapy, however, post-COVID thrombogenesis can also occur at a later stage, in the absence of viral load. The prothrombotic mechanisms related to SARS-CoV-2 differ with regard to the use of antiplatelet and anticoagulant drugs in patients with suspected or confirmed COVID-19. The pharmacology and clinical applicability of anticoagulant drugs such as heparins and oral anticoagulants will be described in this study. This study is an exploratory study aiming to address issues related to the treatment hitherto recommended for thrombotic events in patients affected by COVID-19.
\end{abstract}

Keywords: SARS-CoV-2; COVID-19; Anticoagulants; Venous thromboembolism.

\title{
Resumen
}

La infección causada por el coronavirus SARS-CoV-2 presenta casos clínicos que van desde asintomáticos hasta graves. Incluso en casos leves, se pueden observar secuelas relacionadas con un estado de hipercoagulabilidad asociado a COVID-19, con una mayor prevalencia de tromboembolismo venoso (TEV). La aparición de TEV es mayor en pacientes, principalmente pacientes hospitalizados en unidades de cuidados intensivos y requiere tratamiento anticoagulante, sin embargo, la trombogénesis post-COVID también puede ocurrir en una etapa posterior, en ausencia de carga viral. Los mecanismos protrombóticos relacionados con el SARS-CoV-2 difieren con respecto al uso de fármacos antiplaquetarios y anticoagulantes en pacientes con COVID-19 sospechado o confirmado. En este estudio se describirán la farmacología y la aplicabilidad clínica de los fármacos anticoagulantes como las heparinas y los anticoagulantes orales. Este estudio es un estudio exploratorio que tiene como objetivo abordar cuestiones relacionadas con el tratamiento hasta ahora recomendado para eventos trombóticos en pacientes afectados por COVID-19.

Palabras clave: SARS-CoV-2; COVID-19; Anticoagulantes; Tromboembolia venosa.

\section{Introdução}

A COVID-19 foi considerada uma pandemia pela Organização Mundial da Saúde (OMS) no dia 11 de março de 2020 e se mantém, ainda em 2021, em alta prevalência em diversos países do mundo. A doença causada pelo coronavírus SARSCoV-2 a princípio foi identificada como uma pneumonia grave de etiologia desconhecida. Contudo a infecção causada por esse vírus apresenta quadros clínicos que variam de assintomáticas a graves. Nesse sentido, o sintoma mais preocupante apresentado pelos acometidos é a dispneia, que faz com que muitos pacientes procurem atendimento hospitalar e necessitem de suporte ventilatório.

Além das mazelas deixadas nos casos mais severos da doença, também vêm sendo observadas sequelas naqueles que manifestaram quadros mais brandos do vírus. Há relatos de pacientes recuperados que apresentaram alterações duradouras nos sistemas neurológico, muscular ou circulatório. Entre as consequências relatadas em longo prazo destacam-se fadiga e fraqueza muscular, efeitos como fibrose pulmonar, trombose, embolia e Acidente Vascular Encefálico (AVE), insuficiência renal crônica e lesões hepáticas, bem como o possível desenvolvimento de doenças neurológicas desencadeadas pela infecção (Rocha, 2020; Estevão, 2020; Souto, 2020).

Estudos observacionais têm descrito um estado de hipercoagulação associado à COVID-19, com a prevalência de Tromboembolismo Venoso (TEV). Esse quadro ocorre principalmente em indivíduos críticos, ainda que haja a utilização de anticoagulação profilática. Entretanto essa relação entre o TEV e as infecções causadas por vírus, como da imunodeficiência humana (HIV), hepatite C e influenza, não é nova, já que fora evidenciada na literatura médica (Marques, Sá, Lupi, Brasil, \& Ristow, 2017; Ramacciotti et al., 2019). Sob essa perspectiva, a COVID-19 tem chamado a atenção de angiologistas e cirurgiões vasculares, pois os sintomas estão associados à inflamação do sistema vascular e à hipercoagulabilidade que desencadeiam manifestações clínicas como vasculite de pequenos vasos e trombose micro e macrovascular de artérias e/ou 
veias (Huang et al., 2020). Sendo assim, considerando que eventos trombóticos estão associados à maior mortalidade, torna-se importante o entendimento do TEV pós-COVID-19 para melhor manejo farmacológico durante o período de internação e após alta hospitalar, além da reavaliação periódica dos riscos e benefícios desta abordagem.

\section{Metodologia}

O presente trabalho trata-se de um estudo descritivo exploratório, do tipo transversal, com abordagem qualitativa. É descritivo quando o pesquisador busca retratar a realidade como ela é, sem se preocupar em modificá-la. Todavia tem caráter avaliativo para facilitar a compreensão do assunto em pauta.

Uma vez delimitado o tema da investigação, realizou-se um estudo exploratório, a fim de investigar o tratamento preconizado, no período de fevereiro a maio de 2021, para eventos trombóticos dos pacientes acometidos pela COVID-19.

A coleta de dados se deu por pesquisa em bancos de dados eletrônicos e fontes digitais, como artigos, livros e revistas científicas. A busca limitou-se aos seguintes descritores: SARS-CoV-2, coronavírus, COVID-19 e farmacologia do COVID19, publicados em língua portuguesa e inglesa, no período de fevereiro a maio de 2021. Dessa busca, emergiram 79 documentos, sendo 47 artigos.

O procedimento seguinte consistiu em uma leitura analítica, cuja finalidade foi ordenar as informações contidas nas fontes, possibilitando a obtenção de respostas. Como critério de inclusão dos artigos, selecionaram-se os que continham a abordagem farmacológica de eventos tromboembólicos, enfatizando a Trombose Venosa Profunda (TVP) pós-COVID-19, tema desta pesquisa. Os artigos selecionados advêm da base de dados informatizados BVS, Cochrane, SciELO, PubMed e Science Direct e dos sites oficiais do Ministério da Saúde e de universidades brasileiras e internacionais.

\section{Resultados e Discussão}

\section{A Fisiopatologia Da Trombose}

A trombose é considerada um processo patológico de ativação e propagação imprópria da resposta hemostática do organismo. Caracteriza-se pela formação de trombo na luz do vaso em leito venoso, caso o coágulo se desenvolva em uma veia, ou arterial, se estiver em uma artéria (Simões \& Pinto, 2014).

Os subtipos da trombose permitem a classificação do trombo de acordo com quatro características. A primeira é a estrutura, podendo ser trombos vermelhos, ricos em hemácias, comuns em veias; brancos, que são compostos majoritariamente por plaquetas e fibrinas, típicos das artérias; hialinos, predominantemente de fibrina, tais como em capilares; e mistos, que são os mais comuns, formados por estratificação fibrinosas e cruóricas. A segunda consiste em sua localização, constituindo trombos venosos, cardíacos, arteriais ou capilares. A terceira característica é o efeito de interrupção do fluxo sanguíneo, sendo oclusivos, murais, parietais ou semiocludentes e canalizados. E a última refere-se à presença de infecção, em que o trombo pode ser séptico ou asséptico (Smith, 2021; Grossman, \& Porth, 2015).

A hemostasia, em contrapartida, é caracterizada pelo estado normal do fluxo laminar do sangue, mantido pela harmonia entre forças antitrombóticas e pró-trombóticas, fundamental para o controle de eventos hemorrágicos ou de processos tromboembólicos (Miranda, 2015). O equilíbrio do tampão hemostático pode ser perturbado por três principais anormalidades descritas na teoria da Tríade de Virchow. Nela, lesões endoteliais, distúrbios do fluxo sanguíneo, como a estase sanguínea, e estado de hipercoagulabilidade sanguínea convergem para o desenvolvimento de eventos trombóticos (Mesquita Junior et al., 2013).

O endotélio vascular, que consiste em uma camada de células que reveste a parede interna dos vasos sanguíneos, em situações fisiológicas, participa da homeostase vascular ao promover alterações funcionais adaptativas. Em tais modificações, 
Research, Society and Development, v. 11, n. 1, e12311124991, 2022

(CC BY 4.0) | ISSN 2525-3409 | DOI: http://dx.doi.org/10.33448/rsd-v11i1.24991

as células endoteliais atuam secretando substâncias pró ou anticoagulantes que oportunizam a adesão celular e os mecanismos vasoativos (Bahia et al. 2004). Esse endotélio, entretanto, quando lesado, expõe a matriz extracelular subendotelial e o fator de Von Willebrand, o qual funciona como uma ponte de adesão entre o colágeno subendotelial e o receptor plaquetário GpIIb (Berger et al. 2014). Esse processo de ativação endotelial induz à produção de quantidades maiores de fatores pró-coagulantes, como moléculas de adesão plaquetárias, e teciduais, além de sintetizar menores quantidades de moléculas anticoagulantes, como trombomodulina, PGI2 e t-PA (Kumar et al., 2016).

Sob condições ideais de fluxo sanguíneo laminar normal, plaquetas e outras células do sangue são encontradas no centro do lúmen do vaso, separadas do endotélio por uma camada plasmática em movimento lento (Alves et al., 2015). Quando o fluxo sanguíneo é alterado, observam-se os fenômenos de turbulência, caracterizados pela perda do fluxo linear, que contribui, sobretudo, para a formação de trombose arterial e cardíaca.

Outra possível alteração é a estase, na qual ocorre eventual estagnação completa do fluxo sanguíneo. Esse evento é, pois, um importante elemento formador de trombose venosa (Baruzzi et al., 1996). Ambos os fatores contribuem para a ativação de células endoteliais, induzindo ao aumento da atividade pró-coagulante. Porém a estase, por representar uma diminuição acentuada na velocidade do fluxo sanguíneo, favorece o contato de plaquetas e leucócitos com o endotélio vascular, bem como torna mais lenta a eliminação dos fatores de coagulação ativados, por impedir o influxo de inibidores dos fatores de coagulação (Kumar et al., 2016).

A hipercoagulabilidade sanguínea é o aspecto mais comumente associado aos riscos de desenvolvimento da trombose venosa e, por definição, é uma alteração de ordem primária ou secundária que modifica o equilíbrio das vias de coagulação (Kumar et al., 2016). Nesse caso, os trombos formados são compostos por elementos figurados correlacionados à fibrina e podem originar-se nas artérias, nas veias, no coração e na microcirculação. Os trombos arteriais, por sua vez, desenvolvem-se em locais de pressão elevada e fluxo turbilhonado, sendo constituídos por plaquetas e fibrina. Por fim, os venosos formam-se em regiões de estase e são ricos em hemácias e fibrinas e pobres em plaquetas (Baruzzi et al., 1996).

A ocorrência de TVP pode evoluir para um quadro pós-trombótico, em que há deslocamento de um êmbolo para outros sítios anatômicos. O evento ocasionado por esse desvio é a Embolia Pulmonar (EP), principal causa de óbitos evitáveis em leitos hospitalares (Engelhorn et al., 2002).

Os trombos venosos se formam, em geral, em locais onde a circulação sanguínea é mais lenta, a exemplo dos vasos distais com tendência a se estenderem em direção ao fluxo. Este, se diminuído, favorece a adesão de hemácias e fibrina à parede do vaso, formando um trombo (Alves et al., 2015). Após a obstrução venosa, se os trombos se desprenderem da parede vascular podem ser transportados em direção ao coração e à artéria pulmonar, com consequente embolização, agravando o quadro do paciente (Albuquerque \& Vidal, 1996). No entanto os trombos podem sofrer lise por meio da ativação de fatores fibrinolíticos, os quais permitem a recuperação da função do vaso, ou podem ser resistentes a essa ativação e causar uma obstrução parcial ou completa da veia. Por fim, os trombos podem se reorganizar e se tornar vascularizados, ocorrendo uma recanalização que origina o lúmen (Piccinato, 2008).

A localização do trombo apresenta importância clínica, visto que a manifestação venosa ocorre tanto em veias superficiais quanto em veias profundas do membro inferior. O primeiro caso é sintomático, formando-se na junção safenofemoral e demonstrando baixa chance de embolização (Alves et al., 2015). Já o segundo acontece frequentemente na região do joelho, nas veias poplítea, femoral ou ilíaca. Esse último, em geral, é assintomático e, quando se manifesta clinicamente, através de Tromboembolismo Pulmonar (TEP), pode ser fatal (Baruzzi et al., 1996).

\section{Cascata de coagulação}

A cascata de coagulação envolve uma sequência de processos de ativação proteolítica que leva à formação de 
trombina, a qual está envolvida na proteólise da cascata. Ela pode ser dividida em duas vias: extrínseca e intrínseca. Contudo ambas convergem para um ponto comum a partir da ativação do fator X (Guerra, 1968).

A via extrínseca utiliza elementos sanguíneos e que vão além do espaço intravascular. Ela se inicia em uma lesão tecidual de modo que haja secreção do Fator Tecidual (FT), também conhecida como tromboplastina, a partir das células endoteliais. A tromboplastina, por sua vez, irá ativar o fator VII (FVII), produzindo um complexo FVII ativado/FT (FVIIa/FT). Esse complexo será responsável pela ativação do fator X (Guerra, 1968).

Já a via intrínseca recebe esse nome porque todos os seus componentes estão presentes na circulação sanguínea (Ferreira et al., 2010). Nela, será necessária apenas uma superfície celular de carga negativa para interação com o fator XII (FXII), de Hangeman, o qual, por sua vez, é ativado em fator XIIa pelo seu cofator, o Cininogênio de Alto Peso Molecular (CAPM). O CAPM é responsável por converter a pré-calicreína (PC) em calicreína, que acelera a ativação do FXII. O FXIIa converterá o fator XI em fator XIa, resultando na ativação do fator IX (FIXa). O FIXa, juntamente com o fator VIIIa e com os íons $\mathrm{Ca}^{2+}$, dará origem ao complexo tenase intrínseco, cujo papel é realizar a clivagem do fator X em fator Xa (FXa) (Rodrigues et al., 2012).

Em suma, o FXa, em conjunto com um complexo de íons cálcio, uma superfície de fosfolipídios e a presença do fator $\mathrm{V}$ ativado (ativado pela trombina), é capaz de converter a protrombina (FII) em trombina (FII ativado). A trombina irá atuar na conversão de fibrinogênio em fibrina. Esta última, por outro lado, em conjunto com o complexo fator XIIIA e os íons $\mathrm{Ca}^{2+}$, realiza uma ligação cruzada, gerando o coágulo de fibrina insolúvel (Rodrigues et al., 2012).

Dessa maneira, alguns fatores da cascata de coagulação serão alvos de fármacos, os quais serão abordados posteriormente com maior detalhamento. Vale ressaltar que há fármacos inibidores diretos e indiretos do FXa; os que impedem a conversão de FII em FIIa; os que impossibilitam a síntese dos fatores dependentes de vitamina K; e fármacos que são inibidores diretos da trombina. Dessa forma, utilizam-se esses medicamentos com o intuito de evitar alguns mecanismos trombóticos em pacientes confirmados ou com suspeita de COVID-19. A Figura 1 retrata a cascata de coagulação e os locais de ação de fármacos anticoagulantes. 
Figura 1: Cascata de coagulação e pontos de atuação dos principais fármacos anticoagulantes.

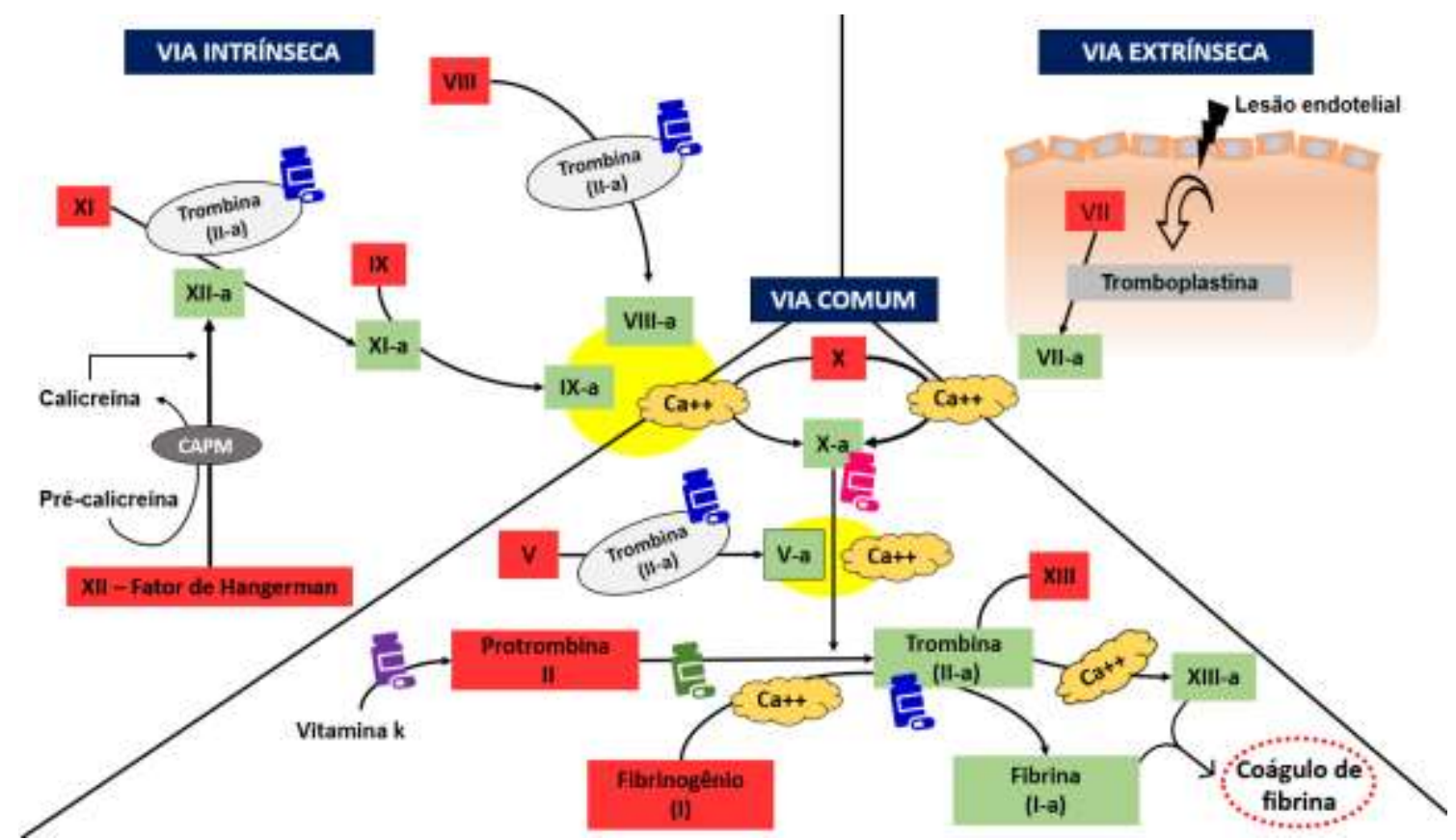

Legenda:

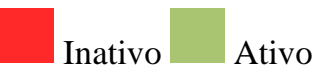

Inibidores diretos e indiretos do FX-a

Inibidores diretos da trombina

Impedem a síntese dos fatores dependentes de vitamina $\mathrm{K}$

Impedem a conversão do FII (protrombina) em FIIa (trombina)

Fonte: Kumar et al. (2016), adaptado pelos autores.

\section{A Trombose Venosa Profunda}

A Trombose Venosa Profunda (TVP) é causada pela formação de trombos nas veias profundas, localizadas nos músculos e ao longo dos ossos. Sua ocorrência é mais comum na parte inferior do corpo, mas pode afetar a veia cava, as veias jugulares internas e a circulação dos membros superiores. Caracteriza-se clinicamente por dor espontânea ou à palpação da perna ou da coxa, inchaço da perna, do pé e/ou do tornozelo, vermelhidão e/ou mudança do tom da pele e aumento da temperatura do membro ou da área afetada.

Os trombos, nesse sentido, provocam oclusão parcial ou total do sistema venoso profundo. Portanto a complicação imediata mais grave associada à TVP é a Embolia Pulmonar (EP), que acontece após o desprendimento de um trombo e a consequente obstrução do fluxo sanguíneo na artéria pulmonar, ocasionando eventos cardiorrespiratórios (Farhat et al. 2018).

A TVP proximal acomete as veias ilíaca, femoral e poplítea. A trombose distal, por sua vez, atinge as veias localizadas abaixo da poplítea. Entretanto o risco de EP e Síndrome Pós-Trombótica (SPT) causada por TVP proximal é maior. 
Research, Society and Development, v. 11, n. 1, e12311124991, 2022

(CC BY 4.0) | ISSN 2525-3409 | DOI: http://dx.doi.org/10.33448/rsd-v11i1.24991

Em contrapartida, existe a possibilidade de desenvolvimento de trombose distal que afeta o segmento proximal em até $20 \%$ das ocorrências, o que demanda o diagnóstico e o tratamento de ambos os casos como TVP proximal (Miranda, 2015).

Os fatores de risco associados ao desenvolvimento de TVP podem ser classificados como hereditários e idiopáticos ou adquiridos. Os primeiros são representados pela resistência à proteína $\mathrm{C}$ ativada, tendo como causas mutação no fator $\mathrm{V}$ de Leiden e no gene da protrombina G20210A, deficiência de antitrombina, de proteína C e de proteína S, alta homocisteinemia e aumento do fator VIII e do fibrinogênio. Já os adquiridos têm como exemplo a presença da síndrome do anticorpo antifosfolipídeo, câncer, hemoglobinúria paroxística noturna, obesidade, gravidez e puerpério, doenças mieloproliferativas, síndrome nefrótica, hiperviscosidade - como macroglobulinemia de Waldenström e mieloma múltiplo -, doença de Behçet, trauma, cirurgias, imobilização e terapia estrogênica (Miranda, 2015).

Os dados epidemiológicos sobre o desenvolvimento de Tromboembolismo Venoso como um evento pós-COVID-19 ainda são de difícil acesso, porém alguns trabalhos estabeleceram essa relação por meio de estudos retrospectivos. Dessa forma, uma pesquisa conduzida no Hospital Israelita Albert Einstein analisou prontuários de 484 casos confirmados de COVID-19. Desses, 13 apresentaram Tromboembolismo Venoso concomitante, em grande maioria, ao regime de terapia intensiva (Cunha et al., 2020). Sendo assim, a prevalência de TEV nesses pacientes hospitalizados foi de 2,7\%.

Outro estudo realizado na Universidade Federal do Espírito Santo avaliou 41 pacientes internados com a forma grave da COVID-19, entre os quais sete apresentaram Tromboembolismo Venoso (Bernardi, et al. 2021). Apesar de pouco abrangentes, os dados sugerem que a investigação de TEV nos pacientes com COVID-19 torna-se imprescindível para o adequado tratamento precoce, a fim de evitar desfechos fatais.

\section{Eventos trombóticos pós-COVID-19}

O agravamento dos pacientes acometidos pela COVID-19 está diretamente associado ao aumento de doenças cardiovasculares, sobretudo de TEV (Huang et al., 2020). Isso porque a liberação em excesso de citocinas como IL-6 leva a uma resposta inflamatória exacerbada e, por conseguinte, ao aumento do risco de produção de trombos (Tang, Li, Wang, \& Sun, 2020). Existe, ainda, a suspeita de que componentes genéticos colaboram para uma maior propensão de desenvolvimento de TEV (Zhou, et al. 2020). Nesse esteio, identificou-se um número surpreendente de eventos trombóticos reportados em virtude da COVID-19, uma vez que a TVP foi detectada por ultrassom Doppler em 15\% dos pacientes que apresentaram quadro de pneumonia associado à presença de D-dímero elevado (Ahmed et al., 2020).

Embora a relação entre a elevação de D-dímero - produto da degradação da fibrina, substância produzida pelo organismo quando há tentativas de desfazer coágulos - e a COVID-19 seja relevante, ela não é exclusiva, uma vez que a elevação desse marcador já foi descrita em outras doenças virais com zika e chikungunya (Ramacciotti et al. 2019).

O endotélio íntegro e funcional é formado por uma camada de glicocálix e secreta fatores ativadores de plasminogênio tecidual, moléculas que impedem a ligação das plaquetas ou bloqueiam o início da cascata de coagulação (Ahmed et al., 2020). Estudos demonstraram, contudo, que o SARS-CoV-2 tem a capacidade de invadir as células endoteliais, gerando lesões e disfunção da parede endotelial vascular e, dessa maneira, predispondo à formação de trombos (Ackermann et al. 2020). Em resposta à invasão viral, a disfunção endotelial estimula o desenvolvimento da trombose por meio da ativação de um estado hiperinflamatório. Nesse cenário, há a liberação de citocinas inflamatórias, como IL-6, TNF- $\alpha$, IL-2, entre outras (Zhang et al., 2017), o que pode levar à síndrome de tempestade de citocinas.

A trombogênese pós-COVID-19 pode ocorrer em uma fase tardia da doença, quando não há mais presença viral, porém ainda se observa disfunção endotelial com perda de glicocálix e da integridade celular (Ahmed et al., 2020). O fluxo sanguíneo encontra-se anormal em razão do aumento da viscosidade sanguínea pela hiperativação do sistema imune, associado às altas concentrações de fibrinogênio e à redução do fluxo microvascular decorrente da hipóxia causada pelos danos 
pulmonares oriundos da infecção. Ademais o estado de hipercoabilidade e hiperviscosidade sanguínea é estimulado pelo desequilíbrio no controle do Sistema Renina-Angiotensina-Aldosterona (SRAA), provocado pela capacidade de o vírus se ligar ao receptor da enzima conversora de angiotensina-2 (ECA-2) presente em células endoteliais de diversos tecidos, sobretudo do tecido pulmonar (Ahmed et al., 2020; Helms et al. 2020; Reno et al., 2015).

A formação de microtrombos e a angiogênese reacional estimulada pelo processo inflamatório caracterizam o distúrbio microvascular de diversos órgãos. Elas, associadas às anormalidades do fluxo sanguíneo, contribuem ainda mais para a lesão endotelial vascular (Bray et al., 2020).

A expressão de elementos teciduais que funcionam como iniciadores da cascata de coagulação, por serem receptores para o fator de coagulação VIII, é estimulada por citocinas inflamatórias como TNF-alfa, endotoxinas e interleucinas. A liberação de histamina, TNF- $\alpha$, IL-8 e IL-6 ainda se correlaciona à maior liberação do fator de Von Willebrand, o que favorece a agregação plaquetária. Além disso, durante a infecção, há elevada produção de fibrinogênio, proteína de fase aguda que perdura na fase pós-COVID-19, aumentando o risco trombótico (Boccia et al., 2020; Gralinski et al. 2018).

O sistema complemento, por sua vez, tem uma participação importante nesse processo, pois manifesta aç̃es próinflamatórias que favorecem a degranulação de mastócitos e o recrutamento de leucócitos na parede vascular, sendo considerado um estímulo à hipercoagulabilidade, conforme citado acima. Logo moléculas do sistema complemento, como a C3a e C5a, relacionam-se também com o aumento da expressão de fatores teciduais e do fator de Von Willebrand, o que beneficia a agregação plaquetária. Do mesmo modo, as proteínas MASP-1 e MASP-2 atuam nesse processo estimulando a conversão da protrombina em trombina e do fibrinogênio em fibrina, o que induz à ativação da cascata de coagulação (Boccia et al., 2020).

O tropismo do SARS-CoV-2, por meio da ligação da proteína spike com a enzima conversora de angiotensina-2 (ECA-2) presente nas células endoteliais, leva a uma desregulação do SRAA (Imai et al. (2005). Esse receptor funciona como um regulador negativo do sistema. Assim não há um controle negativo sobre a ação da angiotensina II (Verdecchia et al., 2020). Estudos demonstram que o bloqueio do receptor ECA-2 está relacionado à formação acentuada de trombos e ao aumento de seu tamanho, enquanto sua função amplificada apresenta efeito antitrombótico (Fraga-Silva et al.). Isso porque a ativação da angiotensina II conduz à produção de inibidores de plasminogênio do tipo 1, ocasionando um estado prótrombótico (Van Leeuwen et al., 1994). Apesar do envolvimento da ECA-2 na ação do vírus SARS-CoV-2, fármacos que inibem o Sistema Renina-Angiotensina-Aldosterona não estão envolvidos na piora do prognóstico da COVID-19. (Vaduganathan, et al. 2020).

\section{Manejo farmacológico da trombose pós-COVID-19}

A farmacologia associada aos mecanismos pró-trombóticos relacionados ao SARS-CoV-2 apresenta divergências no que se refere ao uso de medicamentos antiplaquetários e anticoagulantes em pacientes com suspeita ou infecção confirmada por COVID-19. A heparina, nessa perspectiva, é um dos principais medicamentos de escolha para tratar essa forma mais grave da doença. Sob essa ótica, a farmacologia e a aplicabilidade clínica dos fármacos anticoagulantes - como as heparinas e os anticoagulantes orais - serão descritas a seguir.

\section{Anticoagulantes de uso parenteral}

- $\quad$ Heparina Não Fracionada (HNF) e Heparina de Baixo Peso Molecular (HBPM)

Ao notarem o grande número de eventos trombóticos em pacientes com COVID-19, diversas entidades internacionais criaram diretrizes para a prevenção e o manejo do quadro pró-trombótico dos acometidos pela moléstia. Entre essas instituições, destacam-se a Royal College of Physicians (Spyropoulos et al. 2020) a Anticoagulation Forum (Barnes et al. 
Research, Society and Development, v. 11, n. 1, e12311124991, 2022

(CC BY 4.0) | ISSN 2525-3409 | DOI: http://dx.doi.org/10.33448/rsd-v11i1.24991

2020), a American College of Chest Physicians (Moores et al. 2020), bem como a American Society of Hematology (Izcovich et al. 2020),46, e a International Society of Thrombosis and Haemostasis (ISTH) (Thachil et al. 2020).

Tornou-se consenso o tratamento profilático para Tromboembolismo Venoso em todos os pacientes hospitalizados que não apresentassem contraindicações ao uso dos fármacos. A ISTH (Thachil et al. 2020), por exemplo, recomendou o monitoramento da condição hepática e renal, além dos parâmetros sanguíneos, como D-dímero, tempo de protrombina, contagem plaquetária e níveis de fibrinogênio, uma ou duas vezes ao dia em todos os enfermos. Ademais a ISTH recomendou o uso de Heparina Não Fracionada (HNF), assim como Heparina de Baixo Peso Molecular (HBPM) em todos os pacientes, de forma profilática, até mesmo nos que não apresentavam alterações significativas desses marcadores de coagulação (Moores et al. 2020). A Organização Mundial da Saúde, por outro lado, sugere o uso preferencial de HBPM em dose profilática uma vez ao dia ou, em segundo caso, de HNF por via subcutânea, na dosagem de 5.000 unidades, duas vezes ao dia (World Health Organization, 2020).

A heparina é uma das drogas biológicas mais antigas relacionadas à hemostasia, ao tratamento e à prevenção de Tromboembolismo Venoso (Gray et al., 2008). Sua ação anticoagulante se dá pela ligação com um cofator plasmático que possui a capacidade de levar à inativação da trombina (fator IIa) (Brinkhous et al., 1939). Além do efeito sobre a trombina, a antitrombina III, como foi nomeado esse cofator (Abildgaard, 1968), também possui a capacidade de modular outros fatores de coagulação, intensificando ainda mais sua propriedade antitrombótica (Bussey \& Francis, 2004). Desde que foi iniciado seu uso clínico nos anos 1950 (Theilen, Carr, \& Fowler, 1950; Best, 1959), a heparina permanece como uma das drogas de escolha para intervenção em eventos trombóticos (Kruger et al., 2019; Mazilu et al. 2012). Da mesma forma, existem estudos que mostram que a heparina possui a capacidade de modular a resposta inflamatória, estando envolvida no processo imunológico, na permeabilidade vascular e na proteção endotelial, por exemplo Mazilu et al. (2021).

A Heparina Não Fracionada (HNF) consiste em polímeros lineares que possuem estrutura química heterogênea e, por isso, encontram-se como preparações polidispersas, ou seja, apresentam massas molares variáveis (Mulloy et al., 2000). Apesar dessa característica, os polímeros são formados pela repetição de dissacarídeos trissulfatados, que irão divergir em sua capacidade de ação dependendo de sua composição. As moléculas de heparina de maior potencial anticoagulante exibem um sítio de ligação conservado, cuja constituição é um pentassacarídeo que contém o resíduo 3,6 di-O-sulfated, 2-N-sulfated glucosamine, de vínculação com a antitrombina. Para além desse importante componente, a fim de apresentar sua máxima funcionalidade, é importante que a molécula tenha tamanho suficiente para interagir não somente com a antitrombina, como também com a trombina (fator IIa) e, assim, promover uma ligação covalente entre esses dois compostos (Bussey \& Francis, 2004; Al Dieri et al. 2003; Hirsh et al. 2001).

A HNF, por não ser absorvível de forma eficiente no trato gastrointestinal, deve ser utilizada por via parenteral como injeção subcutânea ou por administração intravenosa (Hull et al. 1986). O fármaco atinge seu pico de atividade muito rapidamente se aplicado dessa forma, possibilitando seu uso quando há necessidade imediata de intervenção. Ademais a droga tem sua meia-vida de 30 a 90 minutos, tornando necessária sua utilização duas vezes ao dia (Nutescu et al., 2016). Convém destacar que a HNF não possui um padrão de ação dose-resposta e está relacionada ao desenvolvimento de trombocitopenia como efeito colateral. Por isso sua utilização deve ser monitorada de forma rigorosa (Bussey \& Francis, 2004; Hull, et al. 1986).

A Heparina de Baixo Peso Molecular (HBPM) começou a ser produzida nos anos 1970, a partir da quebra enzimática da heparina não fracionada (Hao et al., 2019). O objetivo era criar moléculas que apresentassem o sítio pentassacarídico de ligação com a antitrombina III e possuíssem melhores características farmacológicas, possibilitando uma utilização facilitada e mais segura dessa classe de anticoagulantes (Nutescu et al., 2016; Fareed et al. 2003). Desse modo, as HBPMs, como a enoxaparin, dalteparin, tinzaparin e nadroparin, por exemplo, apresentam uma estrutura molecular menor que as Heparinas 
Não Fracionadas. Uma vez que esses fármacos não apresentam a região-C (sequência pentassacarídica + região de ligação com a trombina) em sua composição, a capacidade de modular o fator IIa é reduzida em comparação com as HNFs (Hirsh et al. 2001; McRae et al., 2021), promovendo uma coagulação mais branda nos pacientes e menos efeitos colaterais (McRae et al., 2021).

Essas diferentes HBPMs, para uso clínico, têm características e dosagens próprias, visto que são produzidas de formas distintas e possuem diferenças em sua composição química. Entretanto esses compostos têm um padrão farmacocinético previsível, menor chance de desenvolvimento de trombocitopenia relacionada ao seu uso e tempo de meia-vida mais longo, de três a quatro horas (Tang et al., 2020). Tais atributos possibilitam a aplicação desses fármacos apenas uma vez ao dia, de forma mais segura, o que permite menor monitoramento e utilização fora dos centros médicos (Hirsh et al. 2001; McRae et al., 2021; Hull, et al. 2021).

No caso da ocorrência de sangramentos, estes podem ser revertidos com o emprego da protamina. Contudo, ao contrário de sua eficácia com a HNF, aquela não neutraliza completamente a atividade antiXa da HBPM. Ainda assim, pacientes que sofrem de hemorragia por superdosagem de HBPM devem receber sulfato de protamina ( $1 \mathrm{mg} / 100$ unidades), o que pode reduzir parcialmente o sangramento clínico. Doses menores podem ser suficientes caso a última dosagem de HBPM tenha sido administrada há oito horas ou mais (Bikdeli et al. 2020).

Esse conjunto de fatores faz com que o uso desses fármacos mais modernos torne-se ainda mais interessante, mormente no contexto pandêmico, no qual os serviços de saúde encontram-se em iminência de colapso em diversos países do mundo.

- $\quad$ Inibidores indiretos do fator Xa

O fondaparinux é um anticoagulante sintético baseado na sequência de pentassacarídios da heparina que compõe a região mínima de ligação com a antitrombina (AT), inibindo seletivamente o fator Xa, mas sem afetar a atividade da trombina (Boneu, B., et al. 1995). Apresenta meia-vida de cerca de 18 horas e deve ser administrado por via subcutânea, geralmente em dose única. Mesmo em doses terapêuticas, não altera o PTTa e o Tempo de Protrombina (TP). Desse modo, a monitorização de rotina não é indicada, sendo reservada a dosagem de fator antiXa para ocasiões especiais, como no caso de insuficiência renal (Weitz \& Weitz, 2010). As moléculas muito pequenas de fondaparinux não se ligam à protamina, já que esta é contraindicada para reversão da anticoagulação (Nagler et al., 2012). Atualmente, não há antídoto eficaz para o fondaparinux (Gerotziafas et al., 2004).

Já o idraparinux é um pentassacarídio sintético, para uso parenteral, semelhante ao fondaparinux, do qual se diferencia pelo tempo de ação. Os pentassacarídios foram sintetizados em laboratório por meio da menor partícula da heparina que se ligava à ATIII. Eles se ligam à ATIII e, por conseguinte, ao FXa, formando um complexo terciário irreversível, que, por sua vez, interfere na ação e na formação do complexo protrombinase (Flato et al., 2011).

Após a formação do complexo terciário, o pentassacáridio pode ser liberado desse complexo e inibir nova molécula de ATIII livre no plasma ou aderir a um novo complexo protrombinase. Idraparinux é um anticoagulante de uso subcutâneo, podendo ser administrado a cada sete dias (Flato et al., 2011).

\section{Anticoagulantes orais}

- $\quad$ Antagonista da vitamina K - Varfarina

A varfarina é o anticoagulante oral mais usado na prática clínica ambulatorial. Embora seja amplamente utilizada, não apresenta manejo clínico simples, uma vez que possui estreita janela terapêutica, alta variabilidade na relação dose-resposta e grande interação com outras drogas e a alimentação. A varfarina age a partir da inibição da síntese hepática dos fatores 
dependentes da vitamina K e dos fatores de coagulação II, VII, IX e X. Sua monitorização se faz por meio do exame do Tempo de Protrombina (TP) ou do Tempo de Atividade da Protrombina (TAP) e seu derivado índice internacional normalizado, também conhecido como razão normalizada internacional (INR), sendo mantido entre 2,0 e 3,0 para a maioria das suas indicações. A complicação mais grave provocada por esse fármaco é a hemorragia (Whitlock et al., 2012).

Os pacientes idosos (maiores de 65 anos) são mais sensíveis à medicação, portanto é necessária uma dose diária menor. O sangramento tende a ocorrer com mais frequência nos primeiros três meses de terapia. Com menor frequência, pode ocorrer a necrose da pele e do subcutâneo. Por fim, outras reações adversas incluem hipersensibilidade, icterícia colestática, hepatite, vasculites, náuseas e vômitos, diarreia e alopecia (Levine et al., 2004).

- $\quad$ Inibidores diretos orais do fator Xa (IDFXa)

Os inibidores diretos orais do fator Xa antitrombóticos ligam-se diretamente ao fator Xa sem a necessidade de participação da antitrombina III. A atividade antitrombótica desses agentes é específica para esse fator, sem qualquer interação ou efeito sobre outros fatores da via intrínseca/extrínseca da coagulação e sem resultados indesejáveis, como a trombocitopenia. Tais compostos têm como principais vantagens o baixo peso molecular, a forma de administração oral e sua capacidade de inativar formas circulantes e ligadas do fator Xa (complexo protrombinase $\mathrm{Xa} / \mathrm{Va}$ ). Por apresentarem farmacocinética previsível, o monitoramento de rotina da coagulação não é necessário. Esses fármacos possuem a capacidade de inibir o fator Xa no complexo protrombinase, assim como coágulos ligados a ele, exercendo maior controle sobre a formação e a progressão de trombos, o que lhe confere uma maior eficácia clínica (Flato et al., 2011).

Apixabana é um inibidor oral do fator Xa que impede a atividade do fator livre ou ligado à plaqueta de forma seletiva e reversível, tornando-se capaz de bloquear a atividade do complexo protrombinase. O medicamento apresenta metabolização hepática, meia-vida plasmática de oito a 15 horas e excreção renal e fecal. É um potente IDFXa, com rápida absorção pelo trato gastrointestinal, eliminação renal de $25 \%$ a $30 \%$ e hepática de $65 \%$. Alimentos não interferem na absorção dessa droga. No entanto, assim como o rivaroxabana, a ser abordado, é metabolizado pela enzima CYP3A4, que altera sua disponibilidade na presença de indutores ou inibidores dessa enzima (Flato et al., 2011).

A edoxabana, por sua vez, também é um inibidor oral do fator Xa que se liga, de forma reversível, ao sítio de atividade desse fator. Além disso, é um inibidor competitivo e direto do FXa, com meia-vida de seis a 12 horas e metabolismo predominantemente hepático. Possui as mesmas características de interação farmacológica dos inibidores e dos indutores da CYP3A4 (Flato et al., 2011).

Outro inibidor oral do fator Xa é a rivoraxabana. Composto derivado da oxazolidiona, ela se conecta de forma reversível ao sítio de atividade do fator. Tem posologia única diária, possui rápida absorção e meia-vida de sete a 11 horas e não necessita de ajuste para peso ou sexo. Sua eliminação é feita em $66 \%$ por via hepática e em $33 \%$ por via renal. Em casos de eventos hemorrágicos, descontinuar imediatamente o uso da droga e utilizar complexos de protrombina ativada para reverter quadros graves que apresentam risco de vida (Brandão et al., 2018).

Por fim, a betrixabana também constitui um inibidor direto do FXa, que possui meia-vida de 19 horas, metabolismo predominantemente hepático e excreção biliar. O betrixaban é o único IDFXa para o qual foi desenvolvido um antígeno específico (Kucher et al. 2004). Assim como os Inibidores Diretos da Trombina (IDTs), os IDFXa não possuem um antídoto específico para quadros de sangramento, superdosagem ou necessidade de intervenção cirúrgica não eletiva (Flato et al., 2011).

- Inibidores Diretos da Trombina (IDTs)

Os IDTs, como a dabigatrana, bloqueiam a atividade da trombina em dois sítios: livre no plasma e ligada ao trombo. Logo impedem a conversão do fibrinogênio em fibrina, interferindo nas fases de amplificação e propag ação do modelo celular 
da coagulação pela diminuição da geração de trombina. Além disso, têm como vantagem a propriedade de não se ligarem às proteínas plasmáticas. Sendo assim, os IDTs apresentam uma estabilidade plasmática constante e dispensam a necessidade de monitoração laboratorial em pacientes com clearance de creatinina superior a $30 \mathrm{ml} / \mathrm{kg} / \mathrm{minuto}$, inclusive em obesos ou idosos. Assim os IDTs possuem um rápido pico de ação, eliminação predominantemente renal e não são neutralizados pelo fator plaquetário Tipo 4 (FPT4) (Flato et al., 2011).

A dabigratana é um pró-fármaco que foi lançado há pouco tempo no mercado brasileiro. Ela é rapidamente metabolizada pelo fígado, transformando-se em um composto ativo que se liga de forma competitiva e reversível ao sítio de atividade da trombina, bloqueando sua ação pró-coagulante (Brandão et al., 2018). É um IDT de uso oral e eliminação renal, cuja meia-vida dura entre 12 e 17 horas. Possui biodisponibilidade de 6\%, atingindo, após uma dose oral, o pico de concentração máxima cerca de duas horas depois. Do mesmo modo, apresenta pouca interação com as drogas que envolvem o citocromo P450 (Flato et al., 2011).

\section{Conclusão}

É consenso que pacientes com COVID-19 que não foram internados e que fazem uso prévio de antitrombóticos não necessitam suspender sua utilização. Outrossim devem ser orientados para possíveis interações medicamentosas, com antivirais, anti-inflamatórios hormonais ou não hormonais, tal como identificado nesta pesquisa. Não existe fármaco profilático para pacientes não internados. Todavia a utilização de HBPM pode ser uma ferramenta segura para enfermos com fatores de risco para o desenvolvimento de TVP (Nagler et al., 2012).

A prática de prevenção para Trombose Venosa Profunda para pacientes internados em Unidade de Terapia Intensiva (UTI) é utilizada desde que não haja contraindicação absoluta. No caso de doentes com elevado risco de desenvolver TVP, é recomendada a dose aumentada ou, até mesmo, dobrada de profilaxia durante a internação com a continuidade da farmacoprofilaxia por até 30 dias após a alta do paciente. Cabe ressaltar que essa conduta, nos casos de pessoas hospitalizadas por COVID-19, não encontra embasamento em estudos clínicos ou protocolos, e, portanto, cada paciente deve ser avaliado de forma específica conforme seu quadro clínico (Nagler et al., 2012).

Caso o paciente, durante a internação, seja diagnosticado com TEV, deve-se manter a anticoagulação plena por, pelo menos, três meses. Na alta hospitalar, no contexto desse paciente, recomenda-se farmacoprofilaxia por pelo menos seis semanas (Gerotziafas et al., 2011). De maneira geral, os grupos de enfermos com fatores de risco para trombose merecem um olhar mais atento, em virtude do potencial de apresentação de formas mais graves de trombose.

É de suma importância destacar que é possível prevenir a trombose tanto por meio de medidas não farmacológicas e quanto farmacológicas, tais como citadas ao longo deste estudo. A modalidade não farmacológica é indicada para todas as pessoas. Consiste, pois, em evitar a imobilização prolongada, adotar um estilo de vida saudável, evitar o sedentarismo, manter um peso adequado e, por fim, usar meias elásticas compressivas, este último em caso de indicação médica.

O National Institutes of Health (NIH) dos Estados Unidos (Ferreira et al., 2010), traçou recomendações sobre a associação entre TEV e COVID-19. Segundo o órgão, convém lembrar que, em pacientes não hospitalizados, os quais representam a grande maioria dos casos, não há benefício para o monitoramento de qualquer parâmetro laboratorial em termos de coagulação. Nesse caso, vale considerar a sugestão da American Society of Hematology de que os pacientes podem ser instruídos sobre os sintomas de TEV e, caso identifiquem algum deles, busquem ajuda médica.

Finalmente, estudos futuros específicos sobre a duração precisa da tromboprofilaxia na COVID-19 e em casos de comorbidades específicas devem emergir na literatura científica, visando complementar as diretrizes atuais e contribuir para o manejo farmacológico da TVP na COVID-19. 
Research, Society and Development, v. 11, n. 1, e12311124991, 2022

(CC BY 4.0) | ISSN 2525-3409 | DOI: http://dx.doi.org/10.33448/rsd-v11i1.24991

\section{Referências}

Abildgaard, U. (1968). Highly purified antithrombin III with heparin cofactor activity prepared by disc electrophoresis. Scand J Clin Lab Invest., 21(1), 89-91.

Ackermann, M., Verleden, S. E., Kuehnel, M., Haverich, A., Welte, T., Laenger, F. et al. (2020). Pulmonary Vascular Endothelialitis, Thrombosis, and Angiogenesis in COVID-19. N Engl J Med, 383(2), 120-128.

Ahmed, S., Zimba, O., \& Gasparyan, A. Y. (2020). Thrombosis in Coronavirus disease 2019 (COVID-19) through the prism of Virchow's triad. Clin Rheumatol., 39(9), 2529-2543.

Albuquerque, H. P. C. \& Vidal, C. P. (1996). Trombose Venosa Profunda: Revisão Dos Conceitos Atuais. Rev Bras Ortop, $31(10)$, 851-856.

Al Dieri, R., Wagenvoord R., Van Dedem, G.W.K., Béguin S., \& Hemker, H. C. (2003). The inhibition of blood coagulation by heparins of different molecular weight is caused by a common functional motif-the C-domain. J Thromb Haemost., 1(5), 907-914.

Alves, C. P., Almeida, C. C. \& Balhau, P. A. (2015). Tromboembolismo Venoso Diagnóstico e Tratamento. Sociedade Portuguesa de Cirurgia.

Bahia, L., Aguiar, L., Villela, N. R., Bottino, D., \& Bouskela, E. (2004). Endotélio e aterosclerose. Revista da SOCERJ, 17(1), 26-36.

Barnes, G. D., Burnett, A., Allen, A., Blumenstein, M., Clark, N. P., Cuker, A., Dager, W. E., Deitelzweig, S, B., Ellsworth, S., Garcia, D., Kaatz, S., \& Minichiello, T. (2020). Thromboembolism and anticoagulant therapy during the COVID-19 pandemic: interim clinical guidance from the anticoagulation forum. J Thromb Thrombolysis, 50(1), 72-81.

Baruzzi, A. C. do A., Nussbacher, A., Lagudis, S., \& Souza, J. A. M. (1996). Trombose Venosa Profunda. Profilaxia. http://publicacoes.cardiol.br/abc/1996/6703/67030013.pdf

Berger, M., Silva, W. O. B. da, Santi, L., \& Guimarães, J. A. (2014). Hemostasia: uma breve revisão. Rev Cad Pedagógico, 11(1), $140-148$.

Bernardi, J. M., Silveira, G. C., Polese, J.F., Sant Ana, L., Lara, I. C., Turini, E. A. S. et al. (2021). Incidência de eventos tromboembólicos na COVID-19. Brazilian J Infect Dis., 25(S1), 101078.

Best, C. H. (1959). Preparation of heparin and its use in the first clinical cases. Circulation, 19(1), 70-86.

Bikdeli, B., Madhavan, M. V., Jimenez, D., Chuich, T., Dreyfus, I., Driggin, E. et al. (2020). COVID-19 and Thrombotic or Thromboembolic Disease: Implications for Prevention, Antithrombotic Therapy, and Follow-Up: JACC State-of-the-Art Review. J Am Coll Cardiol. 75(23), $2950-2973$.

Boccia, M., Aronne, L., Celia, B., Mazzeo, G. Ceparano, M., D’Agnano, V. et al. (2020). COVID-19 and coagulative axis: review of emerging aspects in a novel disease. Monaldi Arch Chest Dis., 90(2), 271-276.

Brandão, G. M. S, Cândido, R. C. F, Rollo, H. de A., Sobreira, M. L., \& Junqueira, D. R. (2018). Direct oral anticoagulants for treatment of deep vein thrombosis: Overview of systematic reviews. J Vasc Bras. 17(4),310-317.

Bray, M. A., Sartain, S. E., Gollamudi, J., \& Rumbaut, R. E. (2020). Microvascular thrombosis: experimental and clinical implications. Transl Res., 225, 105130 .

Brinkhous, K. M., Smith, H. P., Warner, E. D., \& Seegers, W. H. (1939). The inhibition of blood clotting: an unidentified substance which acts in conjunction with heparin to prevent the conversion of prothrombin into thrombin. Am J Physiol Content, 125(4), 683-687.

Boneu, B., Necciari, J., Cariou, R., Sié, P., Gabaig, A. M., Kieffer, G. et al. (1995). Pharmacokinetics and tolerance of the natural pentasaccharide (SR90107/ORG31540) with high affinity to antithrombin III in man. Thromb Haemost., 74(6), 1468-1473.

Bussey, H., Francis, J. L. (2004). Heparin Overview and Issues. Pharmacotherapy, 24(8 Part 2):103S-107S.

Cunha, M. V. S.; Pinto, C. A. V.; Guerra, J. C. de C.; Tachibana, A.; Cassino, M. F. (2020). Incidência, diagnóstico, tratamento e desfechos de tromboembolia venosa em pacientes com COVID-19 em um hospital. https://sbacvsp.com.br/incidencia-diagnostico-tratamento-e-desfechos-de-tromboembolia-venosa-empacientes-com-COVID-19 -em-um-hospital-quaternario-no-brasil/.

Engelhorn, A. L. V., Garcia, A. C. F., Cassou, M. F., Birckholz, L., \& Engelhorn, C. A. (2002). Profilaxia da trombose venosa profunda-estudo epidemiológico em um hospital escola. J Vasc Bras, 1(2), 97-102.

Estevão, A. (2020). COVID-19. Acta Radiológica Portuguesa, 32(1), 5-6.

Fareed, J., Hoppensteadt, D., Walenga, J., Iqbal, O., Ma, Q., Jeske, W., \& Taqdees, S. (2003). Pharmacodynamic and pharmacokinetic properties of enoxaparin: Implications for clinical practice. Clin Pharmacokinet., 42(12), 1043-1057.

Farhat, F. C. L. G; Gregório, H. C. T.; Carvalho, R. D. P. de (2018). Evaluation of deep vein thrombosis prophylaxis in a general hospital. J. Vasc. Bras., 17(3), 184-192.

Ferreira, C. N., Sousa, M. de O., Dusse L. M. S., \& Carvalho, M. das G. (2010). O novo modelo da cascata de coagulação baseado nas superfícies celulares e suas implicações. Rev Bras Hematol Hemoter., 32(5), 416-421.

Flato, U. A. P., Buhatem, T., Merluzzi, T., \& Bianco, A. C. M. (2011). Novos anticoagulantes em cuidados intensivos. Rev Bras Ter Intensiva, 23(1),68-77.

Fraga-Silva, R. A., Sorg, B. S., Wankhede, M., de Deugd, C., Jun, J. Y., Baker, M. B. et al. (2010). ACE2 activation promotes antithrombotic activity. Mol Med. 16(5-6), 210-215. 
Gerotziafas, G. T., Depasse, F., Chakroun T., Samama, M. M., \& Elalamy, I. (2004). Recombinant factor VIIa partially reverses the inhibitor effect of fondaparinux on thrombin generation after tissue factor activation in platelet rich plasma and whole blood. Thromb Haemost., 91(3), 531-537.

Gralinski, L. E, Sheahan, T. P., Morrison, T. E., Menachery, V. D., Jensen, K. et al. (2018). Complement activation contributes to severe acute respiratory syndrome coronavirus pathogenesis. MBio., 9(5), 1753-1771.

Gray, E., Mulloy, B., \& Barrowcliffe, T. W. (2008). Heparin and low-molecular-weight heparin. Thromb Haemost. 99(5), 807-818.

Grossman, S \& Porth, C. M. (2015). Porth Fisiopatologia. Guanabara Koogan.

Guerra, C. C. (1968). Fisiologia da coagulação. Matern Infanc, 27(4), 291-295.

Hao, C., Sun, M., Wang, H., Zhang, L. \& Wang, W. (2019). Low molecular weight heparins and their clinical applications. Progress in Molecular Biology and Translational Science., 163, 21-39.

Helms, J., Tacquard, C.; Severac, F., Leonard-Lorant, I., Ohana, M., Delabranche, X. et al. (2020). High risk of thrombosis in patients with severe SARSCoV-2 infection: a multicenter prospective cohort study. Intensive Care Med., 46(6),1089-1098.

Hirsh, J., Warkentin, T. E., Shaughnessy, S. G., Anand, S. S., Halperin, J. L., Raschke, R., Granger, C., Ohman, E. M \& Dalen, J. E. (2001). Heparin and lowmolecular-weight heparin: Mechanisms of action, pharmacokinetics, dosing, monitoring, efficacy, and safety. Chest., 119(1 Suppl.), 64S-94S.

Huang, C., Wang, Y., Li, X., Ren, L., Zhao, J., Hu, Y. et al. (2020). Clinical features of patients infected with 2019 novel coronavirus in Wuhan, China. The Lancet. 2395(10223),497-506.

Hull, R. D., Raskob, G. E., Hirsh, J., Jay, R. M., Leclerc, J. R., Geerts, W. H. et al. (1986). Continuous Intravenous Heparin Compared with Intermittent Subcutaneous Heparin in the Initial Treatment of Proximal-Vein Thrombosis. N Engl J Med., 315(18), 1109-1114.

Hull, R. D., Raskob, G. E., Hirsh, J. et al. (1986). Continuous Intravenous Heparin Compared with Intermittent Subcutaneous Heparin in the Initial Treatment of Proximal-Vein Thrombosis. N Engl J Med., 315(18), 1109-1114.

Hull, R. D., Garcia, D. A., Burnett, A. E. et al. (2021). Heparin and LMW heparin: Dosing and adverse effects. https://www.uptodate.com/contents/heparinand-lmw-heparin-dosing-and-adverse-effects.

Imai, Y., Kuba, K., Rao, S., Huan, Y. Guo, F., Guan, B. et al. (2005). Angiotensin-converting enzyme 2 protects from severe acute lung failure. Nature, 436(7047), 112-116.

Izcovich, A.; Cuker, A.; Kunkle, R.; Neumann, J.; Panepinto, J., Menaka, P. et al. (2020). A user guide to the American Society of Hematology clinical practice guidelines. Blood Adv, 4(9), 2095-2110

Kumar, V., Abbas, A. K. \& Aster, J. C. (2016). Robbins Patologia Básica. Guanabara Koogan.

Kumar, V., Abbas, A. K. \& Aster, J. C. (2016). Robbins \& Cotran Patologia: bases patológicas das doenças. Elsevier.

Kruger, P. C., Eikelboom, J. W.; Douketis, J. D. \& Hankey, G. J. (2019). Deep vein thrombosis: update on diagnosis and management. Med J Aust. 210(11), $516-524$.

Kucher, N., Connolly, S., Beckman, J. A., Cheng, L. H., Tsilimingras, K. V.; Fanikos, J., \& Goldhaber, S. Z. (2004). International normalized ratio increase before warfarin-associated hemorrhage: Brief and subtle. Arch Intern Med. 164(19), 2176-2179.

Levine, M. N.; Raskob G., Beyth, R. J., Kearon, C. \& Schulman, S. (2004). Hemorrhagic complications of anticoagulant treatment: The Seventh ACCP Conference on Antithrombotic and Thrombolytic Therapy. Chest., 126, 287S-310S

Marques, M. A., Sá, F. P. A. de, Lupi, O. Brasil, P., \& Ristow, A. (2017). Trombose venosa profunda e vírus chicungunha. J Vasc Bras., 16(1), 60-62.

Mazilu, L., Katsiki, N., Nikolouzakis, T. K., Aslanidis, G. L., Kouretas, D., Tsatsakis, A. et al. (2021). Thrombosis and Haemostasis challenges in COVID-19

- Therapeutic perspectives of heparin and tissue-type plasminogen activator and potential toxicological reactions-a mini review. Food Chem Toxicol., 148, 111974.

Mesquita Junior, N., Kingerski F. N. M., Marioto, G. L., Viegas, F. A. F., Mesquita, S. F. da S., \& Perreto, S. (2013). Prevalência de trombose venosa profunda em paraplégicos de causa traumática. J Vasc Bras., 12(4), 271-277. doi:10.1590/jvb.2013.051.

McGonagle D, Sharif K, O’Regan A, \& Bridgewood C. (2020). The Role of Cytokines Including Interleukin-6 in COVID-19 Induced Pneumonia and Macrophage Activation Syndrome-Like Disease. Autoimmun Rev Actions, 19(6), 102537.

McRae, H. L., Militello, L. \& Refaai, M. A. (2021). Updates in Anticoagulation Therapy Monitoring. Biomedicines, 9(3), 262.

Miranda, F. (2015). Projeto Diretrizes SBACV: trombose venosa profunda diagnóstico e tratamento. https://sbacvsp.com.br/wpcontent/uploads/2016/05/trombose-venosa-profunda.pdf.

Moores, L. K., Tritschler, T., Brosnahan, S., Carrier, M., Collen, J. F., Doerschug, K., Holley, A. B., Jimenez, D., Gal, G. L., Rali, P. \& Wells, P. (2020). Prevention, Diagnosis, and Treatment of VTE in Patients with Coronavirus Disease 2019: CHEST Guideline and Expert Panel Report. Chest., 158(3), 11431163

Mulloy, B., Gray, E., \& Barrowcliffe, T. W. (2000). Characterization of unfractionated heparin: Comparison of materials from the last 50 years. Thromb Haemost, 84(6), 1052-1056. 
Nagler, M., Haslauer, M., \& Wuillemin, W. A. (2012). Fondaparinux - Data on efficacy and safety in special situations. Thromb Res. $129(4), 407-417$.

Noschang, J., Guimarães, M. D., Teixeira, D. F. D., Braga, J. C. D., Hochhegger, B., Santana, P. R. P., Marchiori, E. (2018). Novas técnicas no diagnóstico por imagem do tromboembolismo pulmonar. Radiol Bras. 51(3), 178-186.

Nutescu E. A., Burnett A., Fanikos, J., Spinler, S., \& Wittkowsky, A. (2016). Pharmacology of anticoagulants used in the treatment of venous thromboembolism. J Thromb Thrombolysis, 41(1), 15-31.

Ramacciotti E., Agati, L. B., Aguiar, V. C. R., Wolosker, N., Guerra, João C., \& Almeida, R. P. de (2019). Zika and Chikungunya Virus and Risk for Venous Thromboembolism. Clin Appl Thromb., 25, 107602961882118.

Reno, L. da C., Silva Junior, V. L. da, \& Trevisan, A. M. (2015). Trombose venosa profunda grave associada ao Sars-Cov-2 (COVID-19). http://sbacvrj.com.br/novo/artigo/trombose-venosa-profunda-grave-associada-ao-sars-cov-2-COVID-19/.

Rocha, C. (2020). Quais as possíveis sequelas causadas pelo novo coronavírus. https://www.nexojornal.com.br/expresso/2020/07/22/Quais-as-possíveissequelas-causadas-pelo-novo-coronavírus.

Rodrigues, E. S.; Castilho-Fernandes, A.; Fontes, A. M. (2012). Novos conceitos sobre a fisiologia da hemostasia. Rev da Univ Val do Rio Verde. 10(1), 218233.

Simões, M. S. M. B. \& Pinto De Oliveira, R. P. de (2014). Principais fatores de risco para trombose venosa profunda. https://atualizarevista.com.br/wpcontent/uploads/2014/10/SIMOES-Myriam-Solange-Martins-Bohana-OLIVEIRA-Rubia-Pinto-de.pdf.

Smith, Y. (2021). Types of Thrombosis. https:/www.news-medical.net/health/Types-of-Thrombosis.aspx.

Souto, X. M. (2020). COVID-19: Aspectos gerais e implicações globais. Recital: Revista de Educação e Tecnologia de Almenara, 2(1).

Spyropoulos, A. C., Levy, J. H.; Walter, A., Connors, J. M., Hunt, B., Iba, T. et al. (2020). Scientific and standardization committee communication: clinical guide on the diagnosis, prevention, and treatment of venous thromboembolism in hospitalized patients with COVID-19.J Thromb Haemost., 18(8),1859-1865.

Tang, N., Li, D. Wang, X., \& Sun, Z. (2020). Abnormal coagulation parameters are associated with poor prognosis in patients with novel coronavirus pneumonia. J Thromb Haemost., 18(4), 844-847.

Thachil, J., Tang, N., Gando, S., Falanga, A., Cattaneo, M., Levi, M., Clark, C. \& Iba, T. (2020). ISTH interim guidance on recognition and management of coagulopathy in COVID-19. J Thromb Haemost. 18(5); 1023-1026. doi:10.1111/jth.14810.

Theilen, E. O., Carr, T. L.\& Fowler, W. M. (1950). The effects of intravenous administration of commercial beef heparin on the thrombocyte count and heparin sensitivity in man. J Lab Clin Med. 35(5), 699-702.

Piccinato, C. E. (2008). Postoperative venous thrombosis. Medicina, 41(4), 477-86

Vaduganathan, M., Vardeny, O., Michel, T., McMurray, J. J. V., Pfeffer, M. A., \& Solomon, S. D. (2020). Renin-angiotensin-aldosterone system inhibitors in patients with COVID-19. N Engl J Med, 382, 1653-1659.

Van Leeuwen, R. T., Kol, A., Andreotti, F., Kluft, C., Maseri, A., \& Sperti, G. (1994). Angiotensin II increases plasminogen activator inhibitor type 1 and tissue-type plasminogen activator messenger RNA in cultured rat aortic smooth muscle cells. Circulation. 90(1), 362-368.

Verdecchia, P., Cavallini, C., Spanevello, A., \& Angeli, F. (2020). The pivotal link between ACE2 deficiency and SARS-CoV-2 infection. Eur J of Intern Med, 76, 14-20.

Weitz, D. S. \& Weitz, J. I. (2010). Update on heparin: What do we need to know? Journal of Thrombosis and Thrombolysis. 29 , $199-207$.

Whitlock, R. P., Sun, J. C., Fremes, S. E., Rubens, F. D., \& Teoh, K. H. (2012). Antithrombotic and thrombolytic therapy for valvular disease: Antithrombotic therapy and prevention of thrombosis, 9th ed: American college of chest physicians evidence-based clinical practice guidelines. Chest., 141(2 SUPPL.), e576S-e600S

World Health Organization (2020). Clinical management of severe acute respiratory infection when novel coronavirus (2019-nCoV) infection is suspected. https://www.who.int/docs/default-source/coronaviruse/clinical-management-of-novel-cov.pdf.

Zhang, Q., Lenardo, M. J., \& Baltimore D. (2017). 30 Years of NF- $\kappa$ B: A Blossoming of Relevance to Human Pathobiology. Cell., $168(1-2), 37-57$.

Zhou, F., Yu, T., Du, R., Fan, G., Liu, Y., Liu, Z. et al. (2020). Clinical course and risk factors for mortality of adult inpatients with COVID-19 in Wuhan, China: a retrospective cohort study. Lancet, 395(10229), 1054-1062. 